\title{
Expanding the Targets of Renal Sympathetic Denervation: From Resistant Hypertension to Atrial Fibrillation
}

\section{Spyridon Koulouris*}

II Clinic of Cardiology, Angiology, Pneumology and Intensive Care Medicine, Germany

\begin{abstract}
Atrial fibrillation is the most common cardiac arrhythmia affecting millions of people worldwide. Individuals with atrial fibrillation sustain significant morbidity mainly due to stroke while their mortality risk is twice higher in comparison to those with sinus rhythm. During the recent years radiofrequency ablation has become a standard procedure for the treatment of atrial fibrillation, albeit with moderate efficacy. Among the risk factors of atrial fibrillation, hypertension has a prominent role. Recently Renal Sympathetic Denervation has been proposed as an effective way to control resistant hypertension showing a sustained reduction in blood pressure. Increased sympathetic activity seems necessary to induce and sustain atrial fibrillation. It can also be considered as one of the common pathways connecting hypertension with atrial fibrillation. Given the limitations of the conventional treatment of atrial fibrillation, Renal Sympathetic Denervation has been proposed as a new treatment modality for the management of this common arrhythmia. Hard data are still lacking but the early results are very promising. Two randomized trials are currently conducted and are expected to answer the question whether the targets of Renal Sympathetic Denervation can expand beyond the treatment of resistant hypertension.
\end{abstract}

Keywords: Sympathetic hyperactivity; Radiofrequency ablation; Pulmonary venous isolation

\section{Introduction}

Atrial Fibrillation (AF) is the most common cardiac arrhythmia affecting millions of people worldwide [1]. Individuals with AF sustain signifincant morbidity mainly due to stroke while their mortality risk is twice higher in comparison to those with sinus rhythm [2]. Moreover, AF poses a significant health care cost burden as a cause of repeated hospitalizations and increased rates of disability [3]. Among the risk factors of AF, hypertension has a prominent role. In the ARIC study, elevated blood pressure alone could explain $21.6 \%$ of the incident cases of paroxysmal AF in the general population [4]. Furthermore, in an elderly population with $\mathrm{AF}$, the prevalence of hypertension was found as high as $84 \%$ [5]. Increased sympathetic activity is considered as one of the common pathways connecting hypertension with AF. A new interventional treatment targeting sympathetic hyperactivity has been recently introduced in order to control resistant hypertension [6]. This treatment modality, known as Renal Sympathetic Denervation $(\mathrm{RDN})$, involves the ablation of the renal sympathetic nerves through radiofrequency emitting catheters inserted percutaneously inside the lumen of both renal arteries. The encouraging results from the initially performed studies has motivated clinicians to pursue new targets for this novel method among other conditions closely associated with increased sympathetic activity. Indeed, RDN has demonstrated beneficial effects on sleep apnea [7], glycemic control [8] and arrhythmias [9]. Given the well established relation among sympathetic hyperactivity, hypertension and AF, trials assessing the efficacy of RDN on AF have already been initiated.

\section{Renal Sympathetic Denervation for the Treatment of Resistant Hypertension}

The role of sympathetic activation in the pathogenesis of cardiovascular diseases has been long recognized and sympathetic nervous system has become a therapeutic target mainly with pharmacological means (i.e beta-blocker administration) [10]. Surgical sympathectomy had also been applied in earlier stages as a more radical way to eliminate symapthetic tone but had never gained popularity among clinicians due to its high incidence of often debilitating side effects (orthostatic hypotension, palpitations, anhydrosis, intestinal disturbances, loss of ejaculation, thoracic duct injuries and atelectasis) [11].

Regarding hypertension, a wealth data support a pathogenetic role for enhanced sympathetic activity. Indeed, surgical attempts to disrupt this hyperactivity have been started in the early decades of the twentieth century [12]. Later on, several pharmacological substances were developed targeting the central or the peripheral divisions of the sympathetic nervous system (ganglionic blockers, a-methyl dopa, clonidine) $[13,14]$. Most of them are still in use. However, during the more recent decades the isight into the mechanisms associated with the renin-agiotensin system had fascinated both researchers and clinicians and the blockers of this pathway had replaced most of the old antisympathetics in clinical practice. Nevertheless, the interest in the role of the sympathetic overflow remained alive in the research field and has regained recently popularity with the development of the so called anti-adrenergic devices.

Sympathetic overflow has been clearly recognized in the majority of the individuals with essential hypertension [15]. This overflow affects mainly the kidneys, the heart and the skeletal muscles. Its remains unclear whether a causal relationship with the development of hypertension exists. Nevertheless, increased sympathetic tone at the level of renal vasculature was been clearly associated with increased

*Corresponding author: Spyridon Koulouris, II Clinic of Cardiology, Angiology Pneumology and Intensive Care Medicine, Klinikum Bayreuth $\mathrm{GmbH}, 101$ Preuschwitzer Str, 95445 Bayreuth, Germany, Tel: 004992115123895; E-mail: spyridon.koulouris@gmail.com

Received July 26, 2013; Accepted August 21, 2013; Published August 23, 2013

Citation: Koulouris S (2013) Expanding the Targets of Renal Sympathetic Denervation: From Resistant Hypertension to Atrial Fibrillation. J Hypertens 2: 123. doi:10.4172/2167-1095.1000123

Copyright: (c) 2013 Koulouris S. This is an open-access article distributed under the terms of the Creative Commons Attribution License, which permits unrestricted use, distribution, and reproduction in any medium, provided the original author and source are credited. 
renin secretion and sodium tubular reabsorption which both play a central role in the pathogenesis of essential hypertension [16]. Moreover, conditions closely associated with essential hypertension like obstructive sleep apnea and insulin resistance seem to depend on sympathetic overflow $[17,18]$. It has been proposed that sub-clincal persistent sympathetic-induced vasoconstriction in the skeletal muscles of patients with essential hypertensions leads directly to reduced glucose uptake, insulin resistance and hyperinsulinemia. On the other hand, sympathetic inbalance due to sleep fragmentation and hypoxic and hypercarbic stimulation of central chemoreceptors in patients with obstructive sleep apnea may consists the main pathogenetic mechanism for the development of hypertension frequently encountered in these patients.

A two-ways relationship seems to exist between kindey function and symathetic overlflow in the hypertensive state. On the one hand sympathetic fibers, wich abudantly innervate both the the kidney vasculature and the nephrons, induce vasoconstriction leading to reduced renal blood flow, increased tubular sodium reabsorption and increased renin secretion rates. On the other hand, kidney baroreceptors and chemoreceptors transmitt afferent stimuli to the central nervous system increasing in this way the sympathetic flow not only back to the kidneys but also to other organ targets like the heart and the peripheral arteries $[19,20]$.

The significance of sympathetic overflow in hypertension has been further supported by the finding of sereval experimental studies which have consistently shown that the interruption of the renal sympathetic nervous system has dramatically reduced the blood pressure in hypertensive animals [21-24].

Percutaneous RDN is a revolutionary new method which based on the above pathophysiological and pre-clinical data, is aiming to become an alternative and effective treatment for hypertensive patients. The Simplicity HTN-1 was the first clinical study to examine the effect of RDN in a cohort of 45 patients which was subsequently expanded to a registry of 153 patients, with the use of a novel percutaneously introduced system [25]. This system uses a catheter to perform endovascularly radiofrequency-ablation of the sympathetic nerves lying within the adventitia of the renal arteries. The radiofrequency energy is been delivered in $90^{\circ}$ quadrants in step-wise fashion to the full circumference of the walls of both renal arteries. All the patients who participated in this trial had resistant hypertension defined as systolic blood pressure $>160 \mathrm{mmHg}$ despite the use of at least 3 antihypertensive medications, including a diuretic. The office systolic and diastolic blood pressures were reduced by $14 / 10 \mathrm{mmHg}$ one month after the procedure, by $27 / 17 \mathrm{mmHg}$ at 12 months and by $33 / 17 \mathrm{mmHg}$ at 36 months. The sympathetic interruption was confirmed by the reduction $(42 \%)$ in renal and total body epinephrine spill over although these measurements were performed in a small number of the above patients [26].

The encouraging results of this prospective study lead to the accomplishment of the first and the only one completed until now randomized trial named Symplicity HTN-2. In this trial 106 patients with resistant hypertension were randomly allocated to renal denervation plus antihypertensive drugs or to antihypertensive drugs alone. At 6-months follow up the intervention group had a reduction in office blood pressure by $32 / 12 \mathrm{mmHg}$ in systolic and diastolic blood pressures respectively [27]. This beneficial effect was sustained at the 12 -months follow up (reduction by $28 / 10 \mathrm{mmHg}$ in systolic and diastolic blood pressures respectively) [28]. In addition, these 2 studies showed that beyond the clinical benefit, the procedure is remarkably safe with local femoral complications and possible renal artery stenosis being the only potential significant hazards.

A recently published systematic review, which included not only the two Symplicity-HTN trials but also several other small series, confirmed the beneficial and sustained effect of RDN in resistant hypertension showing a reduction in systolic and diastolic blood pressures of 18-36 / 9-15 $\mathrm{mmHg}$ respectively [29].

\section{Radiofrequency Ablation for the Treatment of Atrial Fibrillation}

During the recent years radiofrequency ablation has become a standard procedure for the treatment of AF. This treatment modality is largely based on the landmark observation of Hassaguerre et al. that ectopic foci mainly located in the pulmonary veins are responsible for the initiation and the maintenance of the arrhythmia [30]. Circular ablation is Zsx usually performed at the antral area of each individual pulmonary vein aiming to isolate electrically the ectopic foci from the rest of the left atrial myocardium [31]. In addition to this procedure, other ablation techniques have been proposed especially in patients with recurrent or persistent AF. These techniques include the creation of ablation lesions on the left atrial roof and the mitral isthmus [32] as well as, the ablation at sites was highly Complex Fractionated Electrograms (CFAEs) [33] are recorded or even at endocardial sites which correspond to the epicardial location of the ganglionated plexi [34]. Substantial experience has been accumulated during the last decade from the wide clinical application of this method and is as a consequence currently recommended as Class I indication for symptomatic AF refractory to at least one Class I or III antiarrhythmic medication [35]. Nevertheless, its efficacy is rather modest, reported between $66-89 \%$ in the various randomized clinical trials and even lower $55-70 \%$ in the large surveys. Of note, every third patient requires more than one procedure [35]. These data are further confirmed by the recently published head to head comparison of radiofrequency ablation as first line treatment with antiarrhytmic medications in 294 patients with paroxysmal atrial fibrillation [36]. In this trial the total burden of atrial fibrillation did not differ between the two groups at 2 years of follow-up (13\% in the ablation group versus 19\% in the drug group, $\mathrm{p}=0.10)$. Beyond the modest efficacy, the complications rate is also not at all negligible. In the reported surveys complications range from 4.5$6 \%$ with some been serious or even life-threatening (pulmonary vein stenosis, esophageal and phrenic nerve injuries, cardiac tamponade, stroke and silent cerebral microemboli, air embolism and local vascular complications) [37].

\section{Renal Sympathetic Denervation as an Adjunct Treatment in Atrial Fibrillation}

The relatively moderate success of radiofrequency ablation in the treatment of $\mathrm{AF}$ and the highly promising results of the RDN in conjunction with the close association among hypertension, sympathetic hyperactivity and AF, have been the background for a new concept: to apply RDN in the treatment of AF.

The association between AF and sympathetic overflow has long been recognized. B-adrenergic agonists (i.e. isoproterenol) have been successfully used in conjunction with rapid atrial pacing to induce AF in experimental animals [38,39]. Moreover, sympathetic activity seems necessary to sustain AF [40]. It has been postulated that the interference of sympathetic stimulation with intracellular calcium handling may lead to increased calcium concentration with consequent shortening of the action potential and of the refractoriness of ' atrial myocardium 
[41]. This type of "electrical remodeling" is known to predispose to the development of AF. Elegant animal studies have also shown that sympathetic inhibition through RDN may suppress the development of AF induced with rapid atrial pacing [42] or even prevent atrial remodeling after prolonged $\mathrm{AF}$ [43]

Initial data in human studies are also encouraging. Recently, a case was reported were persistent drug-resistant AF was successfully treated with RDN instead of pulmonary venous isolation. Left atrial size was also significantly reduced from $45 \mathrm{~mm}$ to $36 \mathrm{~mm}$ at 6 months of follow-up [44]. A break through trial was also recently reported. In this trial, Pokuschalov et al. [45] showed that pulmonary venous isolation plus RDN was superior to pulmonary venous isolation alone in the prevention of paroxysmal AF in patients with resistant hypertension. From the combined treatment group 9 out of 13 patients $(69 \%)$ were free of episodes at 1 year of follow up, while only 4 out of 14 (29\%) patients were free of episodes in the pulmonary venous isolation alone group.

These data are definitively positive. However, they are still very weak to support of potential anti-arrhythmic role of RDN. Nevertheless, more evidence is expected to come in the near future. The RSD for AF trial is a randomized, controlled multicenter Chinese study expected to give more evidence in this issue [46]. A total of 200 patients with resistant hypertension and symptomatic AF will be assigned to antiarrhythmic and antihypertensive medications or RDN. The burden of AF at 1 year-follow up will be the primary end-point of the study.

Another study, the H-FIB trial [47] will examine the role of adjunctive $\mathrm{RDN}$ at time of $\mathrm{AF}$ ablation. In this multicenter prospective, double-blind, randomized controlled trial patients with hypertension who have their first procedure for the treatment of AF, will be allocated to radiofrequency ablation alone or radiofrequency ablation plus RDN. The end point will be the incidence of drug-free freedom of $\mathrm{AF}$ in the 12 months of follow up.

An argument can be made about the additional benefit that RDN can offer over the administration of beta-blockers in patients with AF. Beta-blockers have long been used for both rate and rhythm control of AF. Their beneficial effect has been proven especially in certain categories of patients like in those with congestive heart failure [48] as well as after cardiac surgery [49], conditions associated with high adrenergic tone. Furthermore, chronic treatment with beta-blockers promotes anti-arrhythmic electrical remodeling of the atria [50]. Nevertheless, a direct comparison between a novel therapy like RDN and a standard treatment modality like beta-blocker administration is unlikely to be performed. Indeed, in the Symplicity HTN trials most of the patients were already taking beta-blockers. In any case, RDN offers the potential to selectively inhibit efferent and afferent sympathetic renal activity without affecting peripheral receptors in the heart and other organs, avoiding this way the negative inotropic and chronotropic effect of beta blockers on the heart as well as their adverse effect on lipid and glucose metabolism.

\section{Conclusions}

The rapidly accumulating positive evidence about the role of RDN in the treatment of hypertension has challenged clinicians who have been tempted to expand its application to other conditions associated with increased sympathetic tone. Atrial fibrillation consist a major and difficult to solve public health problem. The limitations of both antiarrhythmic drugs and left atrial radiofrequency ablation in conjunction with the primary role of sympathetic hyperactivity in the development of this particular arrhythmia have supported the use of RDN in the treatment of AF. It should be acknowledged that the method is new and strong data are lacking. The published results, although encouraging, come from non-randomized trials making the exclusion of a placebo effect impossible. Randomized controlled trials have just started to recruit patients. In addition, only patients with resistant hypertension have been treated so far. Thus, the efficacy of the method in milder types of hypertension and possibly in milder types of sympathetic hyperactivity will remain unknown until the data of new trials come out [51]. Regarding AF, only one non randomized study and a few case reports has been published in the field. Nevertheless, the data are very promising so far. The results of the ongoing trials may eventually offer the evidence which will permit us to use RDN as an adjuvant or event as a first line treatment of AF.

\section{References}

1. Fuster V, Ryden LE, Cannom DS, Crijns HJ, Curtis HJ, Curtis AB, et al. (2006) ACC/AHA/ESC 2006 Guidelines for the Management of Patients with Atria Fibrillation: A Report of the American College of Cardiology/American Heart Association Task Force on Practice Guidelines and the European Society of Cardiology Committee for Practice Guidelines. Circulation 114: e257-e354.

2. Kannel WB, Wolf PA, Benjamin EJ, Levy D (1998) Prevalence, incidence, prognosis, and predisposing conditions for atrial fibrillation: population-based estimates. Am J Cardiol 82: $2 \mathrm{~N}-9 \mathrm{~N}$.

3. Coyne KS, Paramore C, Grandy S, Mercader M, Reynolds M, et al. (2006) Assessing the direct costs of treating nonvalvular atrial fibrillation in the United States. Value Health 9: 348-356.

4. Huxley RR, Lopez FL, Folsom AR, Agarwal SK, Loehr LR, et al. (2011) Absolute and attributable risks of atrial fibrillation in relation to optimal and borderline risk factors: the Atherosclerosis Risk in Communities (ARIC) study. Circulation 123 1501-1508.

5. Piccini JP, Hammill BG, Sinner MF, Jensen PN, Hernandez AF, et al. (2012) Incidence and prevalence of atrial fibrillation and associated mortality among Medicare beneficiaries, 1993-2007. Circ Cardiovasc Qual Outcomes 5: 85-93.

6. Santos M, Carvalho H (2013) Renal sympathetic denervation in resistant hypertension. World J Cardiol 5: 94-101.

7. Guralnick A, Bakris GL (2012) Approaches for targeting blood pressure control in sleep disorders. Curr Opin Nephrol Hypertens 21: 469-474.

8. Thomopoulos C, Spanoudi F, Kyriazis I, Anastasopoulos I, loannidis I (2013) Metabolic effects of renal denervation. Curr Clin Pharmacol 8: 206-211.

9. Ukena C, Mahfoud F, Linz D, Böhm M, Neuberger HR (2013) Potential role of renal sympathetic denervation for the treatment of cardiac arrhythmias. Eurolntervention 9: R110-116.

10. Esler M (2010) The 2009 Carl Ludwig Lecture: Pathophysiology of the human sympathetic nervous system in cardiovascular diseases: the transition from mechanisms to medical management. J Appl Physiol 108: 227-237.

11. Kadowaki MH, Levett JM (1986) Sympathectomy in the treatment of angina and arrhythmias. Ann Thorac Surg 41: 572-578.

12. ALLEN EV (1952) Sympathectomy for essential hypertension. Circulation 6: 131-140.

13. Wiysonge CS, Bradley HA, Volmink J, Mayosi BM, Mbewu A, et al. (2012) Beta-blockers for hypertension. Cochrane Database Syst Rev 8: CD002003.

14. Vongpatanasin W, Kario K, Atlas SA, Victor RG (2011) Central sympatholytic drugs. J Clin Hypertens (Greenwich) 13: 658-661.

15. Esler M, Lambert G, Brunner-La Rocca HP, Vaddadi G, Kaye D (2003) Sympathetic nerve activity and neurotransmitter release in humans: translation from pathophysiology into clinical practice. Acta Physiol Scand 177: 275-284.

16. DiBona GF (2000) Neural control of the kidney: functionally specific renal sympathetic nerve fibers. Am J Physiol Regul Integr Comp Physiol 279: R15171524

17. Witkowski A, Prejbisz A, Florczak E, Kadziela J, Sliwinski P, et al. (2011) Effects of renal sympathetic denervation on blood pressure, sleep apnea course, and glycemic control in patients with resistant hypertension and sleep apnea. Hypertension 58: 559-565. 
Citation: Koulouris S (2013) Expanding the Targets of Renal Sympathetic Denervation: From Resistant Hypertension to Atrial Fibrillation. J Hypertens 2: 123. doi:10.4172/2167-1095.1000123

18. Mahfoud F, Schlaich M, Kindermann I, Ukena C, Cremers B, et al. (2011) Effect of renal sympathetic denervation on glucose metabolism in patients with resistant hypertension: a pilot study. Circulation 123: 1940-1946.

19. DiBona GF, Esler M (2010) Translational medicine: the antihypertensive effect of renal denervation. Am J Physiol Regul Integr Comp Physiol 298: R245-253.

20. Lambert E, Straznicky N, Schlaich M, Esler M, Dawood T, et al. (2007) Differing pattern of sympathoexcitation in normal-weight and obesity-related hypertension. Hypertension 50: 862-868.

21. Kassab S, Kato T, Wilkins FC, Chen R, Hall JE, et al. (1995) Renal denervation attenuates the sodium retention and hypertension associated with obesity. Hypertension 25: 893-897.

22. Campese VM, Kogosov E (1995) Renal afferent denervation prevents hypertension in rats with chronic renal failure. Hypertension 25: 878-882.

23. O'Hagan KP, Thomas GD, Zambraski EJ (1990) Renal denervation decreases blood pressure in DOCA-treated miniature swine with established hypertension. Am J Hypertens 3: 62-64.

24. Ye S, Zhong H, Yanamadala V, Campese VM (2002) Renal injury caused by intrarenal injection of phenol increases afferent and efferent renal sympathetic nerve activity. Am J Hypertens 15: 717-724.

25. Symplicity HTN-1 Investigators (2011) Catheter-based renal sympathetic denervation for resistant hypertension: durability of blood pressure reduction out to 24 months. Hypertension 57: 911-917.

26. Krum H, Schlaich M, Whitbourn R, Sobotka PA, Sadowski J, et al. (2009) Catheter-based renal sympathetic denervation for resistant hypertension: a multicentre safety and proof-of-principle cohort study. Lancet 373: 1275-1281.

27. Symplicity HTN-2 Investigators, Esler MD, Krum H, Sobotka PA, Schlaich MP, et al. (2010) Renal sympathetic denervation in patients with treatmentresistant hypertension (The Symplicity HTN-2 Trial): a randomised controlled trial. Lancet 376: 1903-1909.

28. Esler MD, Krum H, Schlaich M, Schmieder RE, Böhm M, et al. (2012) Renal sympathetic denervation for treatment of drug-resistant hypertension: one-yea results from the Symplicity HTN-2 randomized, controlled trial. Circulation 126 2976-2982.

29. Gosain P, Garimella PS, Hart PD, Agarwal R (2013) Renal sympathetic denervation for treatment of resistant hypertension: a systematic review. J Clin Hypertens (Greenwich) 15: 75-84.

30. Haïssaguerre M, Marcus FI, Fischer B, Clémenty J (1994) Radiofrequency catheter ablation in unusual mechanisms of atrial fibrillation: report of three cases. J Cardiovasc Electrophysiol 5: 743-751.

31. Michaud GF, John R (2011) Percutaneous pulmonary vein isolation for atria fibrillation ablation. Circulation 123: e596-601.

32. Knecht S, Hocini M, Wright M, Lellouche N, O'Neill MD, et al. (2008) Left atrial linear lesions are required for successful treatment of persistent atrial fibrillation. Eur Heart J 29: 2359-2366.

33. Li WJ, Bai YY, Zhang HY, Tang RB, Miao CL, et al. (2011) Additional ablation of complex fractionated atrial electrograms after pulmonary vein isolation in patients with atrial fibrillation: a meta-analysis. Circ Arrhythm Electrophysiol 4 143-148.

34. Pokushalov E, Romanov A, Artyomenko S, Turov A, Shugayev $P$, et al (2010) Ganglionated plexi ablation for longstanding persistent atrial fibrillation. Europace 12: 342-346.

35. Calkins H, Kuck KH, Cappato R, Brugada J, Camm J, et al. (2012) 2012 HRS EHRA / ECAS Expert consensus statement on catheter and surgical ablation of atrial fibrillation: recommendations for patient selection, procedural techniques, patient management and follow-up, definitions, endpoints, and research tria design. J Interv Card Electrophysiol 14: 528-606.

36. Cosedis Nielsen J, Johannessen A, Raatikainen P, Hindricks G, Walfridsson $\mathrm{H}$, et al. (2012) Radiofrequency ablation as initial therapy in paroxysmal atria fibrillation. N Engl J Med 367: 1587-1595.

37. Dagres N, Hindricks G, Kottkamp H, Sommer P, Gaspar T, et al. (2009)
Complications of atrial fibrillation ablation in a high-volume center in 1,000 procedures: still cause for concern? J Cardiovasc Electrophysiol 20: 10141019.

38. Sharifov OF, Fedorov VV, Beloshapko GG, Glukhov AV, Yushmanova AV, et al. (2004) Roles of adrenergic and cholinergic stimulation in spontaneous atrial fibrillation in dogs. J Am Coll Cardiol 43: 483-490.

39. Oral H, Crawford T, Frederick M, Gadeela N, Wimmer A, et al. (2008) Inducibility of paroxysmal atrial fibrillation by isoproterenol and its relation to the mode of onset of atrial fibrillation. J Cardiovasc Electrophysiol 19: 466-470.

40. Jayachandran JV, Sih HJ, Winkle W, Zipes DP, Hutchins GD, et al. (2000) Atrial fibrillation produced by prolonged rapid atrial pacing is associated with heterogeneous changes in atrial sympathetic innervation. Circulation 101 : $1185-1191$.

41. Shen MJ, Choi EK, Tan AY, Lin SF, Fishbein MC, et al. (2011) Neura mechanisms of atrial arrhythmias. Nat Rev Cardiol 9: 30-39.

42. Zhao Q, Yu S, Zou M, Dai Z, Wang X, et al. (2012) Effect of renal sympathetic denervation on the inducibility of atrial fibrillation during rapid atrial pacing. $J$ Interv Card Electrophysiol 35: 119-125.

43. Wang X, Zhao Q, Huang H, Tang Y, Xiao J, et al. (2013) Effect of renal sympathetic denervation on atrial substrate remodeling in ambulatory canines with prolonged atrial pacing. PLoS One 8: e64611.

44. Vollmann D, Sossalla S, Schroeter MR, Zabel M (2013) Renal artery ablation instead of pulmonary vein ablation in a hypertensive patient with symptomatic, drug-resistant, persistent atrial fibrillation. Clin Res Cardiol 102: 315-318.

45. Pokushalov E, Romanov A, Corbucci G, Artyomenko S, Baranova V, et al. (2012) A randomized comparison of pulmonary vein isolation with versus without concomitant renal artery denervation in patients with refractory symptomatic atrial fibrillation and resistant hypertension. J Am Coll Cardiol 60 : 1163-1170.

46. Qiu M, Yin Y, Shan Q (2013) Renal sympathetic denervation versus antiarrhythmic drugs for drug-resistant hypertension and symptomatic atrial fibrillation (RSDforAF) trial: study protocol for a randomized controlled trial. Trials 14:168-174.

47. Ahmed H, Miller MA, Dukkipati SR, Cammack S, Koruth JS, et al. (2013) Adjunctive renal sympathetic denervation to modify hypertension an upstream therapy in the treatment of atrial fibrillation (H-FIB) study: clinical background and study design. J Cardiovasc Electrophysiol 24: 503-509.

48. Nasr IA, Bouzamondo A, Hulot JS, Dubourg O, Le Heuzey JY, et al. (2007) Prevention of atrial fibrillation onset by beta-blocker treatment in heart failure: meta-analysis. Eur Heart J 28: 457-462.

49. Zhu J, Wang C, Gao D, Zhang C, Zhang Y, et al. (2012) Meta-analysis of amiodarone versus $\hat{I}^{2}$-blocker as a prophylactic therapy against atrial fibrillation following cardiac surgery. Intern Med J 42: 1078-1087.

50. Workman AJ, Smith GL, Rankin AC (2011) Mechanisms of termination and prevention of atrial fibrillation by drug therapy. Pharmacol Ther 131: 221-241.

51. Kandzari DE, Bhatt DL, Sobotka PA, O'Neill WW, Esler M, et al. (2012) Catheter-based renal denervation for resistant hypertension: rationale and design of the SYMPLICITY HTN-3 Trial. Clin Cardiol 35: 528-535. 